\title{
Radiation Therapy for Chronic Hidradenitis Suppurativa
}

\section{Samir H Patel ${ }^{1 *}$, Jared R Robbins ${ }^{1}$ and lltefat Hamzavi}

${ }^{1}$ Department of Radiation Oncology, Mayo Clinic, Scottsdale, Arizona, USA

${ }^{2}$ Department of Dermatology, Mayo Clinic, Scottsdale, Arizona, USA

\begin{abstract}
Background: Hidradenitis suppurativa is a chronic follicular occlusive disease in apocrine gland-bearing regions. Frequently refractory to conventional oral and topical treatments, it may require surgical intervention.
\end{abstract}

Objective: To investigate the effectiveness of radiation therapy for refractory hidradenitis suppurativa.

Methods and materials: Five patients with refractory Hurley stage II/III hidradenitis suppurativa were treated with radiation therapy to 13 affected sites. Electron beam radiation to a total dose of 7.5 gray was applied over 3 consecutive days.

Results: The mean age of patients was 45 years; $80 \%$ had hidradenitis suppurativa for $\geq 6$ years. Three patients had Hurley stage III; 2 had Hurley stage II. All had been treated previously with topical and oral antibiotics and other therapies, including surgery, radiation therapy, Nd:YAG laser therapy, and infliximab. No complete responses were observed, but $53 \%$ of the lesions had a partial response. Lesions in the axilla, gluteal, and inguinal areas had response rates of $100 \%, 67 \%$, and $50 \%$, respectively, compared to $0 \%$ for perineal lesions.

Conclusions: Radiation therapy may be a promising treatment for refractory hidradenitis suppurativa. A prospective study is warranted to further evaluate response rates, define optimal dose fractionation schedules, and better understand the risk of long-term toxicity.

Keywords: Inflammatory diseases; Radiotherapy; Disease control

\section{Introduction}

Hidradenitis suppurativa is a disfiguring skin condition characterized by recurring inflammatory and suppurative lesions in the flexural regions (ie, the axillae, inframammary folds, and inguinal, perineal, and intergluteal areas). It can have a tremendously detrimental impact on quality of life [1]. It is a part of the follicular tetrad that also includes acne conglobata, dissecting cellulitis of the scalp, and pilonidal sinus [2]. Follicular hyperkeratosis and occlusion is theorized to be the primary etiologic factor, with apocrine involvement being secondary [3]. Multiple risk factors associated with hidradenitis suppurativa include endocrine causes, obesity, smoking and a genetic component [4]. Epidemiologic data about hidradenitis suppurativa are limited, so its exact prevalence is unknown. Hidradenitis suppurativa is more prevalent in females than in males [5] (4:1 ratio). A primary diagnosis of hidradenitis suppurativa was the presenting complaint for 3,000 patients admitted to the hospital in 2002 in the United States [6].

Hidradenitis suppurativa is grouped into 3 categories by the Hurley staging system [7]. Stage I consists of solitary or multiple isolated abscesses without scarring or sinus tract formation. Stage II is characterized by single or multiple recurrent abscesses with widely separated lesions and sinus tract formation. Stage III is characterized by diffuse involvement across a region with multiple interconnected sinus tracts and abscesses. Treatment is typically categorized as preventive, pharmacologic, surgical, or psychological. A stepwise approach based on the level of evidence for each modality has been reported by Lam et al. [1]. Preventive care includes weight loss, smoking cessation, and avoidance of shaving, avoidance of tight synthetic clothing, stress management, and warm compresses. There is no clear consensus on the use of conservative management, but it may be the most appropriate approach initially for stage I hidradenitis suppurativa. Surgery can play a role in stage I cases in the form of incision and drainage. Beyond conservative management, pharmacologic therapy consists of topical antibiotics, systemic antibiotics, intralesional corticosteroids, hormonal therapy, retinoids, immunosuppressants, and biologic agents [1,4]. The surgical literature suggests that once extensive scarring forms, wide local excision is the treatment of choice $[8,9]$. Promising local therapies include cryotherapy [10], neodymium:yttrium-aluminum-garnet laser therapy $[11,12]$, and radiation therapy $[13,14]$. Furthermore newer therapies such as carbon dioxide laser and the use of anti inflammatory drugs such as infliximab in combination with surgery have shown promise $[15,16]$.

Radiation therapy for treatment of hidradenitis suppurativa was first reported more than 50 years ago [17]. Its use fell out of favor in the 1970s after the development of alternative therapies and because of concern about secondary malignancies and increased costs. However, in the modern era of radiation therapy, some authors have reported on the efficacy of radiation therapy for dissecting cellulitis of the scalp and acne conglobata [18,19]. Frohlich et al. [13] reported complete relief of symptoms in $89(38.5 \%)$ of 231 patients who underwent radiation therapy for hidradenitis suppurativa. More recently, a case report indicated the efficacy of radiation therapy for hidradenitis suppurativa, with a total dose of 7.5 gray given in 2.5-gray fractions [14].

Radiation therapy has the potential to be a viable alternative treatment for chronic hidradenitis suppurativa because of its noninvasive nature. It may also spare patients the toxicity of pharmacologic therapy or the trauma of surgery.

*Corresponding author: Samir $\mathrm{H}$ Patel, Department of Radiation Oncology, Mayo Clinic, 13400 E Shea Blvd, Scottsdale, AZ 85259, USA, E-mail: patel.samir@mayo.edu

Received February 27, 2013; Accepted April 10, 2013; Published April 13, 2013

Citation: Patel SH, Robbins JR, Hamzavi I (2013) Radiation Therapy for Chronic Hidradenitis Suppurativa. J Nucl Med Radiat Ther 4: 146. doi:10.4172/21559619.1000146

Copyright: @ 2013 Patel SH, et al. This is an open-access article distributed under the terms of the Creative Commons Attribution License, which permits unrestricted use, distribution, and reproduction in any medium, provided the original author and source are credited. 
We therefore sought to evaluate the efficacy of radiation therapy for chronic refractory hidradenitis suppurativa.

\section{Methods and Materials}

After obtaining institutional review board approval, we conducted a retrospective review of the institutional database to identify all patients with a diagnosis of Hurley stage II or III hidradenitis suppurativa that had failed to respond to multiple previous therapies. Five patients with hidradenitis suppurativa received radiation treatment to 13 body sites at our tertiary care academic medical institution from October 2010 to March 2011. All patients had been diagnosed more than 5 years previously and all had been treated unsuccessfully with a traditional combination of topical cleansers and topical or oral antibiotics. Three had undergone previous surgery, One had had prior laser treatment, and 1 was receiving immunomodulatory medication. All previous therapies were discontinued at least two weeks prior to initiating radiation therapy.

Baseline data on affected sites, size, drainage, and pain were abstracted from clinical examination records. Simulation was performed on a Varian 2100 series linear accelerator (Varian Medical Systems, Inc, Palo Alto, California) with electron beam radiation therapy capability. Various imaging positions were used, depending on the treatment site. The axillary region was treated with the patient in the supine position. The inguinal and anterior perineal regions were treated with the patient in the frog-leg position. The intergluteal folds were treated with the patient in the prone position. The infragluteal folds and perineal region were treated with the patient in a prone jackknife position. The radiation oncologist clinically outlined the areas of treatment, and customized Cerrobend blocks were created. Patients received radiation treatment to the affected sites to a dose of 7.5 gray in 2.5-gray fractions on consecutive days using an electron beam of 9 million electron volts with a $0.5 \mathrm{~cm}$ bolus prescribed to the $90 \%$ isodose line. Patients were seen in follow-up appointments 2 months after radiation therapy for a clinical examination and were closely followed by their dermatologist. Clinical assessments were made using a subjective global assessment score from the Hidradenitis Suppurativa Foundation, Inc (San Diego, CA).

\section{Results}

Table 1 summarizes the characteristics of these 5 patients. The treated body sites were the perineum (31\%), the axillae (31\%), the infragluteal or buttocks area $(23 \%)$, and the groin $(15 \%)$. The initial response at 2 months as defined by clinical findings and subjective patient response yielded no complete responses, but slightly more than $50 \%$ of the lesions had at least partial improvement. Table 2 details the initial response by treatment site. All the treated axillary areas responded to radiation treatment, whereas no response was observed in any perineal area. The durability of the response was difficult to assess, because most patients went on to receive additional treatments of other types. Table 3 summarizes the treatments each patient received after radiation therapy. Three patients required surgical intervention mostly in the perineal area, 1 was started on infliximab, and 2 were maintained on oral dapsone.

\section{Discussion}

This study is one of the few reports in the English-language medical literature about the use of radiation therapy in the US for hidradenitis suppurativa in the modern era. In these 5 patients, electron beam radiation therapy produced encouraging response rates in the axillae and inguinal folds, with minimal skin toxicity. Previously, a 2010 case

\begin{tabular}{|c|c|c|c|c|c|}
\hline Patient & Age, y/Race/Sex & Hurley Stage & Years Since Diagnosis & Previous Treatment \\
\hline 1 & $43 /$ black/F & III & 24 & Topical and oral antibiotics \\
\hline 2 & $50 /$ black/M & III & 7 & Topical and oral antibiotics; surgery & Infragluteal fold, bilateral axilla, left groin \\
\hline 3 & $51 /$ white/M & III & 20 & $\begin{array}{c}\text { Topical and oral antibiotics; multiple surgeries; } \\
\text { infliximab }\end{array}$ & Infragluteal fold, bilateral anterior perineum, \\
right axilla
\end{tabular}

Abbreviations: F: Female; M: Male; Y: Years

Table 1: Characteristics of 5 patients with chronic refractory hidradenitis suppurativa treated with radiation therapy.

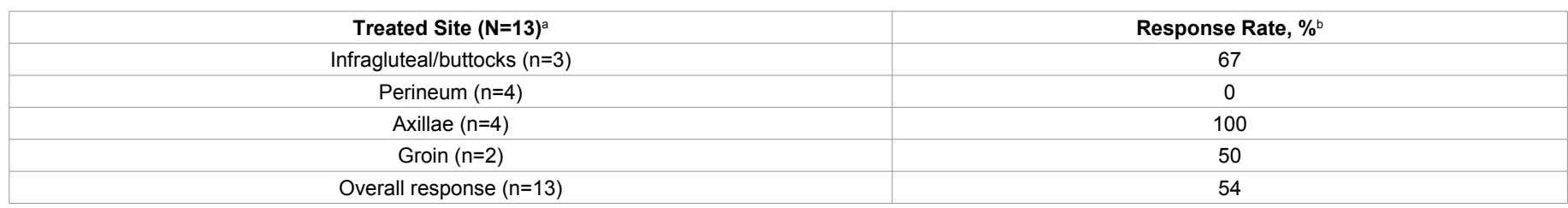

aMost of the 5 patients had more than 1 site affected by hidradenitis suppurativa that was treated with radiation therapy.

${ }^{b}$ Response was defined as clinical findings of and/or subjective report by the patient of at least partial improvement approximately 2 months after completion of radiation therapy

Table 2: Initial Response by Treatment Site to Radiation Therapy in 5 Patients With Chronic Refractory Hidradenitis Suppurativa.

\begin{tabular}{|l|l|}
\hline Patient & Treatment After Radiation Therapy \\
\hline 1 & Oral dapsone; benzoyl peroxide wash; topical clindamycin \\
\hline 2 & $\begin{array}{l}\text { Incision and drainage of lesions in scrotum, perineum, buttock, and left axilla less than } 1 \text { month after radiation therapy; started infliximab } 5 \text { months after } \\
\text { radiation therapy }\end{array}$ \\
\hline 3 & Continued infliximab; surgical débridement of skin of perineum, trunk, and bilateral groin 10 months after radiation therapy \\
\hline 4 & Excision and débridement of soft tissue in right groin with skin graft 3 months after radiation therapy \\
\hline 5 & Oral dapsone \\
\hline
\end{tabular}

Table 3: Further Treatment of Chronic Refractory Hidradenitis Suppurativa After Radiation Therapy. 
report of a patient with primarily axillary and inguinal hidradenitis suppurativa noted a good response to electron beam radiation therapy with no evidence of recurrence at 2.75 years and no evidence of toxicity [14]. Details of the staging of this patient were not available. An earlier series accumulated over a span of 20 years was reported in 2000 by Frolich et al. [13]; of 231 patients treated with radiation therapy in Germany, 89 (38.5\%) showed a complete response rate, whereas 92 (39.8\%) showed some improvement in symptoms. In that report, site-specific data were not available; therefore, it is not clear whether there was a difference in response rates by body site. A very early series reported in 1965 suggested that radiation therapy was efficacious in the axillae [20].

The optimal dose fractionation scheme is not entirely clear and may differ by site. The use of 7.5 gray in 2.5 -gray fractions showed activity in the case reported by Trombetta et al. [14], as well as in our series. A case report of a patient with acquired immunodeficiency syndrome documented resolution of symptoms in the axillae after a single fraction of 4 gray [21]. Frolich et al. [13] used a variety of fractionation schedules (for a total dose of 3-8 gray using 0.5- to 1.5-gray fractions). It is not clear whether escalating the dose would result in improved response rates. Evaluation in a prospective fashion is necessary to better elucidate optimal fraction schedules.

The lack of response to radiation therapy in the perianal/perineal region may be multifactorial. These patients had Hurley stage III hidradenitis suppurativa with significant scarring of the perineum. The use of electron beam radiation targeting the skin may not deposit enough radiation dose at a sufficient depth to significantly affect the scarred tissue. Another theory is that an escalated dose may be required at these sites. One case report of acne conglobata [19] treated with radiation therapy using a fractionation schedule of 20 gray in 2.5gray fractions showed promising results. Another possibility is that advanced perianal/perineal hidradenitis suppurativa is unresponsive to radiation therapy because of the presence of extensive scar tissue. Radiation-induced epilation may be unable to clear the nidus of inflammation at later and chronic stages of this disease.

An important issue to discuss with patients and to consider when planning radiation volume is the mutagenic risk of radiation therapy. As a rough risk estimate, one study measured the effective dose of radiation therapy for benign diseases [22]. The effective dose provides an estimate of the stochastic effects of radiation, which include the carcinogenic and hereditary effects. For a female hidradenitis suppurativa patient receiving 6 gray of radiation to the groin, the effective dose was 16 milliSieverts, with the largest contributions from the colon (12 milliSieverts), skin (3 milliSieverts), and ovary (0.7 milliSieverts). The risk of fatal tumor induction is 2 per 1,000 treated female patients [22].

It is difficult to draw any definite conclusions from our findings because of the retrospective nature and small sample size of this study. Differing patient characteristics and previous treatments can introduce significant bias. However, there may be a role for radiation therapy in chronic hidradenitis suppurativa, particularly in patients with axillary disease. A prospective study is necessary to further evaluate response rates, define optimal dose fractionation schedules, and better understand the risk of long-term toxicity.

\section{References}

1. Lam J, Krakowski AC, Friedlander SF (2007) Hidradenitis suppurativa (acne inversa): management of a recalcitrant disease. Pediatr Dermatol 24: 465-473.

2. Plewig G, Kligman AM (1975) Acne: morphogenesis and treatment. SpringerVerlag, Berlin.

3. Sellheyer K, Krahl D (2005) "Hidradenitis suppurativa" is acne inversa! An appeal to (finally) abandon a misnomer. Int J Dermatol 44: 535-540.

4. Shah N (2005) Hidradenitis suppurativa: a treatment challenge. Am Fam Physician 72: 1547-1552.

5. Galen W, Cohen I, Roger M, Smith M (1995) Bacterial infections: Pediatric dermatology. (2ndedn), Churchill Livingstone, New York.

6. Jemec GB (2003) Hidradenitis suppurativa. J Cutan Med Surg 7: 47-56.

7. Hurley HJ (1989) Axillary hyperhidrosis, apocrine bromhidrosis, hidradenitis suppurativa, and familiar benign pemphigus: surgical approach. Dermatologic surgery: principles and practice. Dekker, New York.

8. Tanaka A, Hatoko M, Tada H, Kuwahara M, Mashiba K, et al. (2001) Experience with surgical treatment of hidradenitis suppurativa. Ann Plast Surg 47: 636-642.

9. Rompel R, Petres J (2000) Long-term results of wide surgical excision in 106 patients with hidradenitis suppurativa. Dermatol Surg 26: 638-643.

10. Bong JL, Shalders K, Saihan E (2003) Treatment of persistent painful nodules of hidradenitis suppurativa with cryotherapy. Clin Exp Dermatol 28: 241-244.

11. Tierney E, Mahmoud BH, Hexsel C, Ozog D, Hamzavi I (2009) Randomized control trial for the treatment of hidradenitis suppurativa with a neodymiumdoped yttrium aluminium garnet laser. Dermatol Surg 35: 1188-1198.

12. Mahmoud BH, Tierney E, Hexsel CL, Pui J, Ozog DM, et al. (2010) Prospective controlled clinical and histopathologic study of hidradenitis suppurativa treated with the long-pulsed neodymium:yttrium-aluminium-garnet laser. J Am Acad Dermatol 62: 637-645.

13. Frohlich D, Baaske D, Glatzel M (2000) Radiotherapy of hidradenitis suppurativa: still valid today? Strahlenther Onkol 176: 286-289.

14. Trombetta M, Werts ED, Parda D (2010) The role of radiotherapy in the treatment of hidradenitis suppurativa: case report and review of the literature. Dermatol OnlineJ 16: 16 .

15. Madan V, Hindle E, Hussain W, August PJ (2008) Outcomes of treatment of nine cases of recalcitrant severe hidradenitis suppurativa with carbon dioxide laser. Br J Dermatol 159: 1309-1314.

16. Van Rappard DC, Mekkes JR (2012) Treatment of severe hidradenitis suppurativa with infliximab in combination with surgical interventions. $\mathrm{Br} J$ Dermatol 167: 206-208

17. Lauge-Hansen N, Lyndrup S (1949) Hidrosadenitis axillaris and its roentgen treatment; optimum total dose and intenseness. Acta Radiol 31: 129-144.

18. Chinnaiyan P, Tena LB, Brenner MJ, Welsh JS (2005) Modern external beam radiation therapy for refractory dissecting cellulitis of the scalp. $\mathrm{Br} \mathrm{J}$ Dermato 152: $777-779$.

19. Myers JN, Mason AR, Gillespie LK, Salkey KS (2010) Treatment of acne conglobata with modern external beamadiation. J Am Acad Dermatol 62: 861 863.

20. Zeligman I (1965) Temporary x-ray epilation therapy of chronic axillary hidradenitis suppurativa. Arch Dermatol 92: 690-694.

21. Johnson SA Jr, Forbes RC (1994) Hidradenitis in a patient with AIDS: palliation with superficial radiation. South Med J 87: 549 .

22. Jansen JT, Broerse JJ, Zoetelief J, Klein C, Seegenschmiedt HM (2005) Estimation of the carcinogenic risk of radiotherapy of benign diseases from shoulder to heel. Radiother Oncol 76: 270-277. 